\title{
Article
}

\section{Antimagicness of subdivided fans}

\author{
Afshan Tabassum ${ }^{1, *}$, Muhammad Awais Umar ${ }^{2}$, Muzamil Perveen ${ }^{1}$ and Abdul Raheem ${ }^{3}$ \\ 1 Department of Mathematics, NCBA \& E, Quaid-e-Azam Campus, Township Lahore, Pakistan.; \\ afshintabassum@gmail.com(A.T); jimi4224@gmail.com(M.P) \\ 2 Govt. Degree College (B), Sharqpur Sharif, Pakistan.; owais054@gmail.com \\ 3 Department of Mathematics, National University of Singapore, Singapore.; rahimciit7@gmail.com \\ * Correspondence: afshintabassum@gmail.com
}

Received: 17 November 2019; Accepted: 10 January 2020; Published: 9 February 2020.

\begin{abstract}
A graph $\Gamma$ (simple, finite, undirected) with an $\Omega$-covering has an $(\alpha, \delta)$ - $\Omega$-antimagic labeling if the weights of all subgraphs $\Omega$ of graph $\Gamma$ constitute an arithmetic progression with the common difference $\delta$. Such a graph is called super $(\alpha, \delta)$ - $\Omega$-antimagic if $v(V(\Gamma))=\{1,2,3, \ldots,|V(\Gamma)|\}$. In the present paper, the cycle coverings of subdivision of fan graphs has been considered and results are proved for several differences.
\end{abstract}

Keywords: $\Omega$-covering, super $(\alpha, \delta)$ - $\Omega$-antimagic graph, cycle-antimagic, super cycle-antimagic, fan graphs.

MSC: 05C78, 05C70.

\section{Introduction}

$\mathbf{L}$ et $\Gamma=(V(\Gamma), E(\Gamma))$ be a finite simple and undirected graph with a family of subgraphs $\Omega_{1}, \Omega_{2}, \ldots, \Omega_{t}$ such that every element of $E(\Gamma)$ belongs to $\Omega_{i} \cong \Omega, i=1,2, \ldots, t$, then $\Gamma$ admits an $\Omega$-covering. An $\Omega$-covered graph $\Gamma$ with $v$ is called an $(\alpha, \delta)$ - $\Omega$-antimagic if $w t_{v}(\Omega)=\{\alpha, \alpha+\delta, \ldots, \alpha+(t-1) \delta\}$ where the associated $\Omega$-weights denoted by $w t_{v}(\Omega)$ are defined as

$$
w t_{v}(\Omega)=\sum_{v \in V(\Omega)} v(v)+\sum_{e \in E(\Omega)} v(e) .
$$

and $\alpha>0$ and $\delta \geq 0$ are two integers, $t$ is the number of $\Omega_{i} \cong \Omega$. For a total labeling $v$ to be super we require $v(V(\Gamma))=\{1,2, \ldots,|V(\Gamma)|\}$.

The results about $\Omega$-(super)magic graphs with $\Omega$ as cycle, path and tree can be studied in [1-7].

Inayah et al. [8] introduced the $(\alpha, \delta)$ - $\Omega$-antimagic labeling. We refer [9-11] for some results on super $(\alpha, \delta)$ - $\Omega$-antimagic labeling. In [12], Lih proved that $F_{n}$ is $C_{3}$-supermagic for every $n$ except $n \equiv 2(\bmod 4)$. In [7], Ngurah et al. proved that $F_{n}$ is $C_{3}$-supermagic for every $n \geq 2$. In the present paper, we proved the super $(\alpha, \delta)-C_{r+2 k+3}$-antimagic labelings of subdivided fans for differences $\delta=0,1,2,3,4$.

\section{Preliminaries}

In this section, we give basic definitions of concepts concerning a subdivided fan $F_{n}(r, k)$.

Definition 1. A graph $F_{n} \cong P_{n}+K_{1}$ is called fan graph obtained by the join of path $P_{n}$ and one isolated vertex $K_{1}$.

The central vertex, or the hub vertex is of degree $n$ and path vertices are the other ones. Spokes are the adjacent edges of central vertex and path edges are the remaining edges.

$$
\begin{aligned}
& V\left(F_{n}\right)=\{c\} \cup\left\{x_{1}, x_{2}, \ldots, x_{n}\right\}, \\
& E\left(F_{n}\right)=\left\{x_{1} x_{2}, x_{2} x_{3}, \ldots, x_{n-1} x_{n}\right\} \cup\left\{c x_{1}, c x_{2}, \ldots, c x_{n}\right\} .
\end{aligned}
$$


Definition 2. The subdivided fan $F_{n}(r, k)$ is the graph obtained from a fan $F_{n}$ by inserting $r \geq 1$ new vertices $\left\{v_{1}^{(i)}, \ldots, v_{r}^{(i)}\right\}$ into each path edge $x_{i} x_{i+1}, 1 \leq i \leq n-1$, denoted by $P_{x_{i}} x_{i+1}$-vertices and by inserting $k \geq 1$ new vertices $\left\{w_{1}^{(i)}, \ldots, w_{k}^{(i)}\right\}$ into every spoke $c x_{i}, 1 \leq i \leq n$, denoted by $S^{(i)}$-vertices.

$$
\begin{aligned}
E\left(P_{x_{i} x_{i+1}}\right) & =\left\{x_{i} v_{1}^{(i)}, v_{2}^{(i)} v_{3}^{(i)}, \ldots, v_{r-1}^{(i)} v_{r}^{(i)}, v_{r}^{(i)} x_{i+1}, 1 \leq i \leq n-1\right\} \\
E\left(S^{(i)}\right) & =\left\{c w_{1}^{(i)}, w_{2}^{(i)} w_{3}^{(i)}, \ldots, w_{k-1}^{(i)} w_{k}^{(i)}, w_{k}^{(i)} x_{i}, 1 \leq i \leq n\right\}
\end{aligned}
$$

Let $C_{r+2 k+3}^{(i)}$ be the $i^{\text {th }}$-subcycle. For the weight of $i^{\text {th }}$-subcycle $C_{r+2 k+3}^{(i)}$, we obtain

$$
\begin{aligned}
w t_{\psi}\left(C_{r+2 k+3}^{(i)}\right)= & \sum_{u \in V\left(C_{r+2 k+3}^{(i)}\right)} \psi(u)+\sum_{e \in E\left(C_{r+2 k+3}^{(i)}\right)} \psi(e) \\
= & \left(\psi\left(x_{i}\right)+\psi\left(x_{i+1}\right)+\psi(c)+\sum_{v \in V\left(P_{\left.x_{i} x_{i+1}\right)}\right.} \psi(v)+\sum_{w \in V\left(S^{(i)}\right)} \psi(w)\right) \\
& +\left(\sum_{e \in E\left(P_{x_{i} x_{i+1}}\right)} \psi(e)+\sum_{e \in E\left(S^{(i)}\right)} \psi(e)+\sum_{e \in E\left(S^{(i+1)}\right)} \psi(e)\right) .
\end{aligned}
$$

where indices $i$ are taken modulo $n$.

\section{Main results}

In this section, we introduce the super $(\alpha, \delta)-C_{r+2 k+3}$-antimagic labelings of subdivided fans for differences $d=0,1,2,3,4$.

Theorem 1. Let $r, k \geq 1$ and $n \geq 3$ be positive integers. The subdivided fan $F_{n}(r, k)$ is super $(\alpha, \delta)$ - $C_{r+2 k+3}$-antimagic for difference $\delta=0,1,4$.

Proof. The total labeling $\psi_{\delta}$ is defined as:

$$
\begin{gathered}
\psi_{\{\delta\}}(c)=1 \\
\psi_{\{0,4\}}\left(x_{i}\right)= \begin{cases}\left\lceil\frac{n}{2}\right\rceil+2-\frac{i+1}{2}, & \text { if } i \equiv 1(\bmod 2) \\
n+2-\frac{i}{2}, & \text { if } i \equiv 2(\bmod 2)\end{cases} \\
\psi_{\{1\}}\left(x_{i}\right)= \begin{cases}1+\frac{i+1}{2}, & \text { if } i \equiv 1(\bmod 2) \\
1+\left\lceil\frac{n}{2}\right\rceil+\frac{i}{2}, & \text { if } i \equiv 2(\bmod 2)\end{cases} \\
\psi_{\{0,1\}}\left(c w_{1}^{(i)}\right)= \begin{cases}2(n-1)(r+1)+2(n k+1)+\frac{i+1}{2}, & \text { if } i \equiv 1(\bmod 2) \\
2(n-1)(r+1)+2(n k+1)+\left\lceil\frac{n}{2}\right\rceil+\frac{i}{2}, & \text { if } i \equiv 2(\bmod 2)\end{cases} \\
\psi_{\{4\}}\left(c w_{1}^{(i)}\right)=2 n(r+k)+(3 n-2 r+1)-i .
\end{gathered}
$$

For $\delta=0,1,4$

$$
\begin{aligned}
\psi_{\delta}\left(V\left(P_{x_{i} x_{i+1}}\right)\right) & =\{(n-1) j+2+i: 1 \leq i \leq n-1,1 \leq j \leq r\} \\
\psi_{\delta}\left(E\left(P_{x_{i} x_{i+1}}\right)\right) & =\{(n-1)(2 r+2-j)+n(k+1)+2-i: 1 \leq j \leq r+1\} \\
\psi_{\delta}\left(V\left(S^{(i)}\right)\right) & =\{r(n-1)+1+n j+i: 1 \leq i \leq n, 1 \leq j \leq k\} \\
\psi_{\delta}\left(E\left(S^{(i)}\right) \backslash\left\{c w_{1}^{(i)}\right\}\right) & =\{2 n(r+k)+(3 n-2 r+1)-n j-i: 1 \leq j \leq k\}
\end{aligned}
$$

where indices $i$ are taken modulo $n$. Evidently $\psi_{\delta}$ is a super labeling as $\left.V\left(F_{n}(r k)\right)\right)=\{1,2, \ldots, n(k+r+1)-$ $r+1\}$. The spoke vertices are labeled with the numbers $n+2, n+3, \ldots, n+2 k+1$ and the path edge vertices are labeled with $n+2 k+2, n+2 k+3, \ldots, n(k+r+1)-r+1$. Clearly, 


$$
\begin{aligned}
\sum\left(\psi_{\delta}\left(V\left(S^{(i)}\right)\right)+\psi_{\delta}\left(E\left(S^{(i)}\right) \backslash\left\{c w_{1}^{(i)}\right\}\right)\right) & =3 r k(n-1)+k(3 n+2 n k+2) \\
\sum\left(\psi_{\delta}\left(V\left(P_{x_{i} x_{i+1}}\right)\right)+\psi_{\delta}\left(E\left(P_{x_{i} x_{i+1}}\right)\right)\right) & =n r(2 r+k+4)+n(k+2)++r(1-2 r)+1-i
\end{aligned}
$$

According to (1) and (2), we obtain

$$
\begin{aligned}
w t_{\psi_{0}}\left(C_{r+2 k+3}^{(i)}\right)= & 4(n-1)(r+1)+4(n k+1)+6 r k(n-1)+2\left\lceil\frac{n}{2}\right\rceil+n+3+2 k(3 n+2 n k+2) \\
& +n r(2 r+k+4)+n(k+2)+r(1-2 r)+1 \\
w t_{\psi_{0}}\left(C_{r+2 k+3}^{(i)}\right)= & n+2\left\lceil\frac{n}{2}\right\rceil+n k(7 r+4 k+11)+2 n r(r+4)+r(1-2 r-6 k)+2(3 n+2 k-2 r)+4 .
\end{aligned}
$$

Equation (3) shows that all $C_{r+2 k+3}^{(i)}$-weights are independent of $i$.

According to (1) and (2), we obtain

$$
\begin{aligned}
w t_{\psi_{1}}\left(C_{r+2 k+3}^{(i)}\right)= & 4(n-1)(r+1)+4(n k+1)+6 r k(n-1)+2\left\lceil\frac{n}{2}\right\rceil+5+2 i+2 k(3 n+2 n k+2) \\
& +n r(2 r+k+4)+n(k+2)+r(1-2 r)+1-i \\
w t_{\psi_{1}}\left(C_{r+2 k+3}^{(i)}\right)= & 2\left\lceil\frac{n}{2}\right\rceil+n k(7 r+4 k+11)+2 n r(r+4)+r(1-2 r-6 k)+2(3 n+2 k-2 r)+6+i .
\end{aligned}
$$

Equation (4) shows that all $C_{r+2 k+3}^{(i)}$-weight consists of consecutive integers.

According to (1) and (2), we obtain

$$
\begin{aligned}
w t_{\psi_{4}}\left(C_{r+2 k+3}^{(i)}\right)= & 4(n-1)(r+1)+4(n k+1)+6 r k(n-1)+2\left\lceil\frac{n}{2}\right\rceil+5+2 i+2 k(3 n+2 n k+2) \\
& +n r(2 r+k+4)+n(k+2)+r(1-2 r)+1-i \\
w t_{\psi_{4}}\left(C_{r+2 k+3}^{(i)}\right)= & n+\left\lceil\frac{n}{2}\right\rceil+r(1-2 r)+k(4 n k-5 r+9)+2 n(3 k+4)(r+1)-6 n+7-4 i .
\end{aligned}
$$

Equation (5) shows that all $C_{r+2 k+3}^{(i)}$-weight constitute an arithmetic progression with common difference $\delta=4$. This completes the proof.

Theorem 2. Let $r, k \geq 1$ and $n \geq 3$ be positive integers. The subdivided fan $F_{n}(r, k)$ is super $(\alpha, \delta)$ - $C_{r+2 k+3}$-antimagic for difference $\delta=2,3,5$.

Proof. The total labeling $\psi_{\delta}$ is defined as:

$$
\begin{gathered}
\psi_{\{\delta\}}(c)=1 \\
\psi_{\{\delta\}}\left(x_{i}\right)=2 i \\
\psi_{\{\delta\}}\left(v_{r}\right)=2 i+1 \\
\psi_{\{3\}}\left(c w_{1}^{(i)}\right)= \begin{cases}2 n(r+k)+(3 n-2 r+1)-\frac{i+1}{2}, & \text { if } i \equiv 1(\bmod 2) \\
2 n(r+k)+(3 n-2 r+1)-\left\lceil\frac{n}{2}\right\rceil-\frac{i}{2}, & \text { if } i \equiv 2(\bmod 2)\end{cases} \\
\psi_{\{5\}}\left(c w_{1}^{(i)}\right)= \begin{cases}2\{r(n-1)+n(k+1)\}+\frac{i+1}{2}, & \text { if } i \equiv 1(\bmod 2) \\
2\{r(n-1)+n(k+1)\}+\left\lceil\frac{n}{2}\right\rceil+\frac{i}{2}, & \text { if } i \equiv 2(\bmod 2)\end{cases}
\end{gathered}
$$

For $\delta=2,3,5$

$$
\begin{aligned}
\psi_{\delta}\left(V\left(P_{x_{i} x_{i+1}}\right)\right) & =\{n+(n-1) j+1+i: 1 \leq i \leq n-1,1 \leq j \leq r-1\} \\
\psi_{\delta}\left(E\left(P_{x_{i} x_{i+1}}\right)\right) & =\{(n-1)(2 r+2-j)+n(k+1)+2-i: 1 \leq j \leq r+1\} \\
\psi_{\delta}\left(V\left(S^{(i)}\right)\right) & =\{r(n-1)+1+n j+i: 1 \leq i \leq n, 1 \leq j \leq k\} \\
\psi_{\delta}\left(E\left(S^{(i)}\right) \backslash\left\{c w_{1}^{(i)}\right\}\right) & =\{2 n(r+k)+(3 n-2 r+1)-n j-i: 1 \leq j \leq k\}
\end{aligned}
$$


where indices $i$ are taken modulo $n$.

Evidently $\psi_{\delta}$ is a super labeling as $\left.V\left(F_{n}(r k)\right)\right)=\{1,2, \ldots, n(k+r+1)-r+1\}$. The spoke vertices are labeled with the numbers $n+2, n+3, \ldots, n+2 k+1$ and the path edge vertices are labeled with $n+2 k+2, n+$ $2 k+3, \ldots, n(k+r+1)-r+1$. Clearly,

$$
\begin{aligned}
\sum\left(\psi_{\delta}\left(V\left(S^{(i)}\right)\right)+\psi_{\delta}\left(E\left(S^{(i)}\right) \backslash\left\{c w_{1}^{(i)}\right\}\right)\right) & =3 r k(n-1)+k(3 n+2 n k+2) \\
\sum\left(\psi_{\delta}\left(V\left(P_{x_{i} x_{i+1}}\right)\right)+\psi_{\delta}\left(E\left(P_{x_{i} x_{i+1}}\right)\right)\right) & =n(r+1)(k+3)+2 n\left(r^{2}-1\right)+r(n-2 r+1)+1 .
\end{aligned}
$$

According to (1) and (6), we obtain

$$
\begin{aligned}
& w t_{\psi_{2}}\left(C_{r+2 k+3}^{(i)}\right)=2 i+3+n\{k(r+5)+7 r+3\}+2 n k(2 k+3)+2 n\left(r^{2}-1\right)+2(3 n-2 r+2 k+1) \\
& w t_{\psi_{2}}\left(C_{r+2 k+3}^{(i)}\right)=n k(4 k+r+11)+n\left(2 r^{2}+7 r+1\right)+2(3 n-2 r+2 k)+5+2 i .
\end{aligned}
$$

Equation (7) shows that all $C_{r+2 k+3}^{(i)}$-weights constitute an arithmetic progression with common difference $\delta=2$.

According to (1) and (6), we obtain

$$
\begin{aligned}
w t_{\psi_{3}}\left(C_{r+2 k+3}^{(i)}\right)= & 6 n-4 r+5+3 i-\left\lceil\frac{n}{2}\right\rceil+r(n-2 r+1)+6 k r(n-1) \\
& +2 k n(2 k+3)+4 k+n\left(2 r^{2}+5 k+7 r+r k+1\right) \\
w t_{\psi_{3}}\left(C_{r+2 k+3}^{(i)}\right)= & 2 n r(r+4)+n k(4 k+r+11)+6 r k(n-1)-r(2 r+3)+7 n+4 k-\left\lceil\frac{n}{2}\right\rceil+5+3 i .
\end{aligned}
$$
$\delta=3$.

Equation (8) shows that all $C_{r+2 k+3}^{(i)}$-weights constitute an arithmetic progression with common difference According to (1) and (6), we obtain

$$
w t_{\psi_{5}}\left(C_{r+2 k+3}^{(i)}\right)=n k(6 r+11)+n r(3 r+8)-r(2 r+3)+2 k(2 n k-3 r+2)+5 n+\left\lceil\frac{n}{2}\right\rceil+5+5 i .
$$
$\delta=5$.

Equation (9) shows that all $C_{r+2 k+3}^{(i)}$-weights constitute an arithmetic progression with common difference

\section{Concluding remarks}

An $\Omega$-covering graphs is the extension of the edge-antimagic labeling and generalizes the structure for $\Omega$-antimagic labeling. Several results concerning $\Omega$-antimagic labelings for different families of graphs are proved and available in literature. In the present manuscript, the super $(\alpha, \delta)-C_{n}$-antimagicness of subdivided fans has been considered for few of differences. One can work to extend the labeling for further differences greater than 5 .

Acknowledgments: The authors are grateful for the valuable comments of the anonymous referees.

Author Contributions: All authors contributed equally to the writing of this paper. All authors read and approved the final manuscript.

Conflicts of Interest: “The authors declare no conflict of interest."

\section{References}

[1] Gutiérrez, A., \& Lladó, A. (2005). Magic coverings. Journal of Combinatorial Mathematics and Combinatorial Computing, 55, 43-56.

[2] Inayah, N., López, A., \& Moragas, J. (2012). Magic and antimagic H-decompositions. Discrete Mathematics, 312(7), 1367-1371.

[3] Jeyanthi, P., \& Selvagopal, P. (2010). More classes of $H$-supermagic graphs. International Journal of Algorithms, Computing and Mathematics, 3(1), 93-108.

[4] Lladó, A., \& Moragas, J. (2007). Cycle-magic graphs. Discrete Mathematics, 307(23), 2925-2933.

[5] Maryati, T. K., Baskoro, E. T., \& Salman, A. N. M. (2008). $P_{h}$-supermagic labelings of some trees. Journal of Combinatorial Mathematics and Combinatorial Computing, 65, 197. 
[6] Maryati, T. K., Salman, A. N. M., \& Baskoro, E. T. (2013). Supermagic coverings of the disjoint union of graphs and amalgamations. Discrete Mathematics, 313(4), 397-405.

[7] Ngurah, A. A. G., Salman, A. N. M., \& Susilowati, L. (2010). H-supermagic labelings of graphs. Discrete Mathematics, 310(8), 1293-1300.

[8] Inayah, N., Salman, A. N. M., \& Simanjuntak, R. (2009). On (a, d)-H-antimagic coverings of graphs. Journal of Combinatorial Mathematics and Combinatorial Computing, 71, 273-281.

[9] Inayah, N., Simanjuntak, R., Salman, A. N. M., \& Syuhada, K. I. A. (2013). Super (a, d)-H-antimagic total labelings for shackles of a connected graph H. Australasian Journal of Combinatorics, 57, 127-138.

[10] Umar, M. A. (2019). Cyclic-antimagic construction of ladders. Engineering and Applied Science Letter, 2(2), $43-47$.

[11] Ali, N., Umar, M. A., Tabassum, N., \& Raheem, L. (2018). Super (a,d)-C3-antimagicness of a corona graph. Open Journal of Mathematical Sciences, 2(1), 371-378.

[12] Lih, K. W. (1983). On magic and consecutive labelings of plane graphs. Utilitas Math, 24, 165-197.

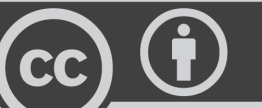

BY

(c) 2020 by the authors; licensee PSRP, Lahore, Pakistan. This article is an open access article distributed under the terms and conditions of the Creative Commons Attribution (CC-BY) license (http://creativecommons.org/licenses/by/4.0/). 